\title{
Channel Estimation Based on the Discrete Cosine Transform Type-III Even
}

\author{
María Elena Domínguez-Jiménez*, David Luengo ${ }^{\dagger}$, Gabriela Sansigre-Vidal* $^{*}$ \\ *Escuela Técnica Superior de Ingeniería Industrial (ETSII), Universidad Politécnica de Madrid, 28006 Madrid (Spain) \\ $\dagger$ Escuela Técnica Superior de Ing. y Sist. de Telecom. (ETSIST), Universidad Politécnica de Madrid, 28031 Madrid (Spain)
}

\begin{abstract}
In this work, we address the problem of channel estimation in multicarrier communications. We present a procedure which employs the Type-III even DCT (DCT3e) at both the transmitter and the receiver. By using any symmetric training symbol we show how to estimate the channel's impulse response without a prior knowledge of its exact length. Theoretical results are provided in order to guarantee the validity of the proposed technique, whereas simulations illustrate the good behavior of the proposed estimation algorithm.

Index Terms-MCM, Channel estimation, DCT.
\end{abstract}

\section{INTRODUCTION}

Many current digital communication systems make use of multicarrier modulation (MCM) techniques. For example, orthogonal frequency division multiplexing (OFDM) techniques employ the discrete Fourier transform (DFT). Discrete cosine transforms (DCTs) have been considered as an alternative to the DFT for MCM schemes due to their good behavior under carrier frequency offset (CFO) [1]-[6]. In any case, MCM systems require the estimation of the channel's impulse response (CIR), which is usually unknown. This estimation is typically achieved by using a known training symbol, and several authors have addressed channel estimation and equalization in OFDM [7]-[10]. Similarly, other works have provided estimation algorithms for different types of DCTs. For instance, in [11] estimation techniques were developed for the Type-II and Type-IV even DCTs; the proposed solutions present good behavior, but they need extra DCTs in order to compute the 1-tap coefficients for channel estimation. Regarding the Type-I even DCT, some estimation algorithms have also been presented: in [12] for symmetric channels and in [13] for any kind of channel, symmetric or not. However, the approach of [13] is not general in the sense that it requires specific training symbols with enough leading and tail zeros. In order to overcome these problems, in the present work we analyze the use of the Type-III even DCT (DCT3e) as an efficient solution.

The paper is organized as follows. In Section II we recall the general formulation of the channel estimation problem, and the DCT3e is introduced: we first provide some properties of the DCT3e in Section II-A, the proposed estimation procedure by using DCT3e is then presented in Section II-B, and in Section II-C we give a simplified version of such procedure for short filters. Section III contains some numerical examples that illustrate the behaviour of our algorithm. Finally, we summarize the conclusions of this work in Section IV.

\section{DCT3E FOR ChANNEl EStimation}

The DCT3e of an $N$-length signal is given by the matrix $\mathbf{C}_{3 e}$ shown in [14], whose entries are

$$
c_{k, j}=2 \alpha_{j} \cos \left(\frac{\pi(2 k+1) j}{2 N}\right) \quad k, j=0, \ldots, N-1,
$$

where

$$
\alpha_{j}= \begin{cases}\frac{1}{2}, & \text { if } j \in\{0, N-1\} \\ 1, & \text { otherwise. }\end{cases}
$$

We consider the general channel estimation problem of Fig. 1: our aim is to estimate the unknown $L$-length channel impulse response, $\mathbf{h}=\left[h_{0}, \cdots, h_{L-1}\right]^{T}$, by means of an $N$-length training symbol $\mathbf{x}$, which is known, and a discrete transform $\mathbf{T}$. The received signal is $\mathbf{y}=\mathbf{x} * \mathbf{h}+\mathbf{z}$, of length $L+N-1$, where $\mathbf{z}$ is a term related to the additive noise and $*$ denotes the standard linear convolution operator. In the receiver, the aim is to estimate $\mathbf{h}$, by means of the 1-tap coefficients $d_{i}$, which are known a priori because they depend on the information in $\mathbf{x}$ and the transform $\mathbf{T}$.

In this work, we will make use of the DCT3e as the transform $\mathbf{T}$ both at the transmitter and the receiver, and a known symbol with whole-sample (WS) symmetry:

$$
\mathbf{x}=\left[x_{M}, \cdots, x_{1}, x_{0}, x_{1}, \cdots, x_{M}\right]^{T} .
$$

In order to present our procedure, we first need to provide some properties of DCT3e.

\section{A. Convolution and MIRAS procedure}

The convolution of the vector $\mathrm{x}$ with any vector (of length $N=2 M+1)$ can be written as the product with the general convolution matrix,

$$
\mathbf{X}_{c o n v}=\left[\begin{array}{rrrr}
x_{M} & 0 & \cdots & 0 \\
\vdots & x_{M} & \ddots & \vdots \\
x_{1} & \ddots & \ddots & 0 \\
x_{0} & \ddots & \ddots & x_{M} \\
\vdots & \ddots & \ddots & \vdots \\
x_{M} & \ddots & \ddots & x_{1} \\
0 & x_{M} & \ddots & x_{0} \\
\vdots & \ddots & \ddots & \vdots \\
0 & \cdots & 0 & x_{M}
\end{array}\right],
$$




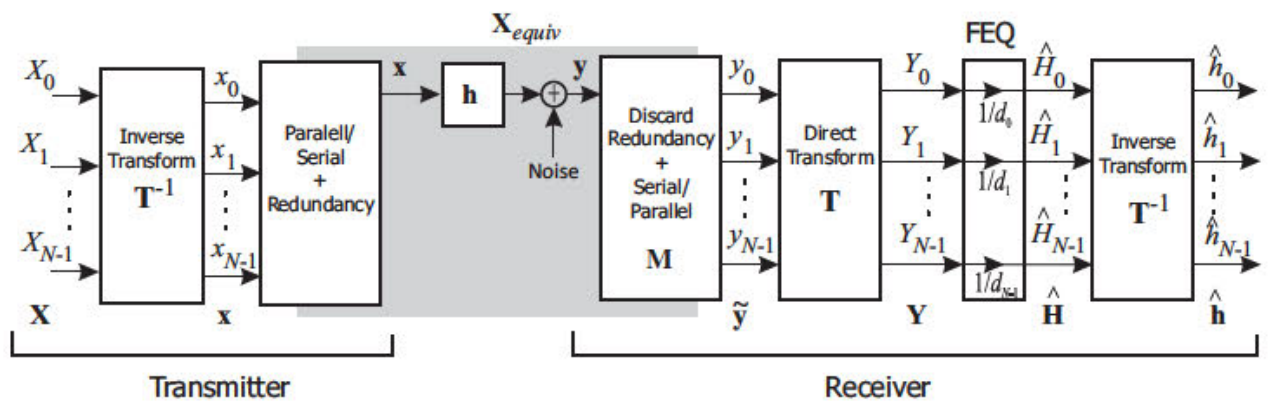

Fig. 1. General block diagram for channel estimation in MCM transceivers.

which has $2 M+N$ rows and $N$ columns. Our first aim is to modify its rows in order to obtain an equivalent $N \times N$ matrix that is perfectly diagonalized by the DCT3e.

For this goal, we need to define the auxiliary matrix $M$, the matrix that transforms an arbitrary vector of length $N+2 M=$ $4 M+1, \mathbf{c}=\left[c_{0}, c_{1}, \ldots, c_{4 M}\right]^{T}$, in the following way:

$$
\begin{aligned}
\mathbf{M} \cdot \mathbf{c}= & {\left[c_{M}, . ., c_{2 M-1}, c_{2 M}, c_{2 M+1}, c_{2 M+2}, \ldots, c_{3 M}\right]^{T} } \\
& +\left[c_{M}, \ldots, c_{1}, \quad 0, \quad 0,-c_{4 M}, . .,-c_{3 M+2}\right]^{T} .
\end{aligned}
$$

Notice that the final vector discards its first component, $c_{0}$, and produces a reversal (mirror symmetry) of the following $M$ components, adding them to the adjacent ones (replicating $c_{M}$ ); it also produces a mirror symmetry of its last $M-1$ components, and substracts them from the previous ones (discarding $\left.c_{3 M+1}\right)$. Therefore, it is a "mirror, replicate, add/subtract" procedure that we call MIRAS. The explicit expression of the $N \times(N+2 M)$ matrix $\mathrm{M}$ is given in Figure 2. Now we are ready to present our main theoretical result:

Proposition 1: Let us consider the convolution matrix $\mathbf{X}_{\text {conv }}$ in (1) and the MIRAS matrix $\mathbf{M}$ shown in Fig. 2. Then, there exists an $N \times N$ matrix $\mathrm{X}_{\text {conv }}$ which is diagonalized by the DCT3e, and that verifies

$$
\mathbf{M} \cdot \mathbf{X}_{\text {conv }} \cdot[0, \mathbf{z}]^{T}=\mathbf{X}_{\text {equiv }} \cdot[0, \mathbf{z}]^{T}
$$

for any vector $\mathrm{z}$ of length $N-1$.

Proof: It suffices to apply the MIRAS procedure to the rows of $\mathbf{X}_{\text {conv }}$ : reversing its first/last rows and adding/substracting them to the adjacent ones, the obtained matrix is $\mathbf{M} \cdot \mathbf{X}_{\text {conv }}$, that can be easily written as

$$
\mathbf{M} \cdot \mathbf{X}_{\text {conv }}=\mathbf{T}+\mathbf{K}^{\prime}
$$

being $\mathbf{T}$ and $\mathbf{K}^{\prime}$, respectively, Toeplitz and Hankel-type matrices of the kind:

$$
\mathbf{T}=\left[\begin{array}{rrrrrrr}
x_{0} & x_{1} & \cdots & x_{M} & 0 & \cdots & 0 \\
x_{1} & \ddots & \ddots & & & \ddots & \vdots \\
\vdots & \ddots & \ddots & \ddots & & & 0 \\
x_{M} & & \ddots & \ddots & & & x_{M} \\
0 & & & & & & \vdots \\
\vdots & \ddots & & & & & x_{1} \\
0 & \cdots & 0 & x_{M} & \cdots & x_{1} & x_{0}
\end{array}\right]
$$

$$
\mathbf{K}^{\prime}=\left[\begin{array}{rccccr}
x_{0} & x_{1} & \cdots & x_{M} & \cdots & 0 \\
\vdots & \vdots & . & 0 & . & \vdots \\
x_{M-1} & x_{M} & . & & . & 0 \\
0 & 0 & . & & . & -x_{M} \\
\vdots & \vdots & . & & . & \vdots \\
0 & 0 & \cdots & -x_{M} & \cdots & -x_{2}
\end{array}\right] .
$$

Theoretical results in [15] guarantee that $\mathbf{T}+\mathbf{K}$ is diagonalized by the DCT3e if $\mathbf{K}=\mathbf{K}^{\prime}$ except for its first column, which is null. We simply make this modification and define the matrix $\mathbf{X}_{\text {equiv }}=\mathbf{T}+\mathbf{K}$, which is diagonalized by the DCT3e and shares all of its columns with $\mathbf{M} \cdot \mathbf{X}_{\text {conv }}$, so the claim holds.

This result is the basis of the proposed estimation procedure that is explained in the following section.

\section{B. Channel Estimation using MIRAS-DCT3e}

In this section, we present our channel estimation technique by means of the DCT3e, and the MIRAS procedure that we have introduced in the previous section. We first choose a WS known symbol x of length $N=2 M+1$. As we are interested in the estimation of the unknown channel filter $\mathbf{h}$ by means of a DCT3e block of size $N$, let us introduce the $N$-length zero-padded version of $\mathbf{h}$ :

$$
\mathbf{h}_{z p}=\left[0, \mathbf{h}^{T}, \mathbf{0}_{N-L-1}\right]^{T},
$$

i.e., $\mathbf{h}_{z p}$ is obtained by appending 1 zero to the left and enough zeros to the right up to length $N$ (this extension is performed because the channel length $L$ is usually smaller than the symbol length $N)$. The $(N+2 M)$-length received vector is then

$$
\mathbf{y}_{z p}=\mathbf{x} * \mathbf{h}_{z p}+\mathbf{z}_{z p}=\mathbf{X}_{\text {conv }} \cdot \mathbf{h}_{z p}+\mathbf{z}_{z p},
$$

with $\mathbf{z}_{z p}$ being the noise vector. We apply the MIRAS procedure to the received vector $\mathbf{y}_{z p}$, as explained in (2) and depicted in Fig. 3, and we obtain the $N$-length vector

$$
\widetilde{\mathbf{y}}=\mathbf{M} \cdot \mathbf{y}_{z p} .
$$

For the estimation procedure, we recall Proposition 1, which ensures that

$$
\begin{aligned}
\widetilde{\mathbf{y}} & =\mathbf{M} \cdot \mathbf{X}_{\text {conv }} \cdot \mathbf{h}_{z p}+\mathbf{M} \cdot \mathbf{z}_{z p} \\
& =\mathbf{X}_{\text {equiv }} \cdot \mathbf{h}_{z p}+\mathbf{M} \cdot \mathbf{z}_{z p},
\end{aligned}
$$




$$
\mathbf{M}=\left[\begin{array}{cccccccc}
0 & \mathbf{0}_{1 \times(M-1)} & 2 & \mathbf{0}_{1 \times(M-1)} & \mathbf{0}_{1 \times 2} & \mathbf{0}_{1 \times(M-1)} & 0 & \mathbf{0}_{1 \times(M-1)} \\
\mathbf{0}_{M \times 1} & \mathbf{J}_{M-1} & \mathbf{0}_{(M-1) \times 1} & \mathbf{I}_{M-1} & \mathbf{0}_{M \times 2} & \mathbf{0}_{M \times(M-1)} & \mathbf{0}_{M \times 1} & \mathbf{0}_{M \times(M-1)} \\
\mathbf{0}_{2 \times 1} & \mathbf{0}_{2 \times(M-1)} & \mathbf{0}_{2 \times 1} & \mathbf{0}_{2 \times(M-1)} & \mathbf{I}_{2} & \mathbf{0}_{2 \times(M-1)} & \mathbf{0}_{2 \times 1} & \mathbf{0}_{2 \times(M-1)} \\
\mathbf{0}_{(M-1) \times 1} & \mathbf{0}_{(M-1)} & \mathbf{0}_{(M-1) \times 1} & \mathbf{0}_{(M-1)} & \mathbf{0}_{(M-1) \times 2} & \mathbf{I}_{M-1} & \mathbf{0}_{(M-1) \times 1} & -\mathbf{J}_{M-1}
\end{array}\right]
$$

Fig. 2. Explicit expression of the MIRAS matrix $\mathbf{M}$, with $\mathbf{I}_{N}$ and $\mathbf{J}_{N}$ denoting $N \times N$ identity and counter-identity matrices, respectively.

where $\mathbf{X}_{\text {equiv }}$ is diagonalized by an $N$-point DCT3e:

$$
\mathbf{C}_{3 e} \cdot \mathbf{X}_{\text {equiv }} \cdot \mathbf{C}_{3 e}^{-1}=\mathbf{D} .
$$

Moreover, [15] guarantees that the diagonal entries of matrix $\mathbf{D}$ (eigenvalues of $\mathbf{X}_{\text {equiv }}$ ) are the DCT3e transform of the vector $\mathbf{x}_{z p}^{r}=\left[x_{0}, \ldots, x_{M}, 0, \ldots, 0\right]^{T}$ :

$$
d_{k}=\left[\mathbf{C}_{3 e} \cdot \mathbf{x}_{z p}^{r}\right]_{k}, \quad k=0, \ldots, N-1 .
$$

Therefore, we are now able to find an easy solution to the channel estimation problem by using the DCT3e: it suffices to denote $\mathbf{Y}:=\mathbf{C}_{3 e} \cdot \widetilde{\mathbf{y}}, \mathbf{H}:=\mathbf{C}_{3 e} \cdot \mathbf{h}_{z p}$, and $\mathbf{Z}:=\mathbf{C}_{3 e} \cdot \mathbf{M} \cdot \mathbf{z}_{z p}$, to obtain

$$
\mathbf{Y}=\mathbf{D} \cdot \mathbf{H}+\mathbf{Z}
$$

The coefficients $d_{k}$ can be computed and stored in memory when choosing a specific training signal $\mathbf{X}$; then, vector $\mathbf{x}$ is easily related to them by defining $\mathbf{X}:=\mathbf{C}_{3 e} \cdot \mathbf{x}$, so $\mathbf{x}$ and $d_{j}$ are also known. Finally, we obtain an estimation of $\mathbf{H}$,

$$
\widehat{\mathbf{H}}_{k}=\mathbf{Y}_{k} / d_{k}, \quad k=0, \ldots, N-1,
$$

and compute $\widehat{\mathbf{h}}_{z p}=\mathbf{C}_{3 e}^{-1} \cdot \widehat{\mathbf{H}}$, which gives a perfect estimation of $\mathbf{h}_{z p}=\left[0, \mathbf{h}^{T}, 0, \ldots, 0\right]^{T}$ in the absence of noise.

\section{Further simplification of the procedure:}

Some of the above computations may be simplified, because the last $2 M-L$ components of $\mathbf{y}_{z p}$ are null:

$$
\mathbf{y}_{z p}=\mathbf{x} *\left[0, \mathbf{h}^{T}, \mathbf{0}_{N-L-1}\right]^{T}+\mathbf{z}_{z p}=\left[0, \mathbf{y}^{T}, \mathbf{0}_{2 M-L}\right]^{T} .
$$

Hence, the MIRAS transform of $\mathbf{y}_{z p}$ does not have to be applied to its last $2 M-L$ components, since their contribution is 0 . Moreover, there are two cases in this simplification:

- If the channel's length $L$ is small enough (i.e., $L \leq M+$ 1 ), then the last $M-1$ components of $\mathbf{y}_{z p}$ are null, since $2 M-L \geq M-1$. Hence, no "mirror and substract" procedure is needed at the last components of $\mathbf{y}$. In this case, we would simply obtain

$$
\begin{aligned}
\widetilde{\mathbf{y}}=\mathbf{M} \cdot \mathbf{y}_{z p}= & {\left[y_{M}, . ., y_{2 M-1}, . ., y_{3 M}\right]^{T} } \\
& +\left[y_{M}, \ldots, y_{1}, 0,0, \cdots, 0\right]^{T} .
\end{aligned}
$$

In other words, it suffices to apply a "mirror, replicate and add" procedure (MIRA) only to the first $M$ components of $\mathbf{y}$ and then apply zero-padding to the right in order to obtain the $N$-length vector $\widetilde{\mathbf{y}}$.

- On the other hand, if $L>M+1$ there are $L-(M+1)$ non-null final components and we will obtain

$$
\begin{aligned}
\widetilde{\mathbf{y}}= & \mathbf{M} \cdot \mathbf{y}_{z p}=\left[y_{M}, . ., y_{2 M-1}, . ., y_{3 M}\right]^{T} \\
& +\left[y_{M}, \ldots, y_{1}, 0, \cdots, 0,-y_{4 M+4-L}, . .,-y_{3 M+2}\right]^{T} .
\end{aligned}
$$

Hence, in this case we only need to apply the "mirror and subtract" to the last $L-(M+1)$ components of $\mathbf{y}_{z p}$.

In any case, the resulting $\widetilde{\mathbf{y}}$ is an $N$-length vector. Figure 3 shows the general MIRAS procedure; but as we have shown, these computations can be eventually simplified if the maximum length of the channel is smaller than $M+1$.

\section{SUMMARY OF THE OPTIMIZED PROCEDURE:}

1) Choose a training signal $\mathbf{X}$ of odd length $N$, such that $\mathbf{x}=\mathbf{C}_{3 e}^{-1} \cdot \mathbf{X}$ is a WS signal.

2) Compute the DCT3e of the right-hand vector of $x$ : $\mathbf{C}_{3 e} \cdot \mathbf{x}_{Z P}^{r}=\mathbf{d}$, and store it in memory.

3) Transmit $\mathbf{x}$ through the channel, obtaining the vector $\mathbf{y}$ at the receiver.

4) Append zeroes to the right of $\mathbf{y}$ and modify it by the MIRAS procedure (mirror the edge components, replicate and add/substract them to/from their adjacent ones), in order to get the vector $\tilde{\mathbf{y}}$ of length $N$ :

- If $L \leq M+1$, simply apply MIRA ("mirror, replicate and add") only to the first $M$ components of $\mathbf{y}$, and then perform zero-padding to obtain an $N$-length vector $\widetilde{\mathbf{y}}$.

- If $L>M+1$, we apply MIRA to the first $M$ components of $\mathbf{y}$ and also apply "mirror and substract" to the $L-(M+1)$ final samples of $\mathbf{y}$, obtaining the $N$-length vector $\widetilde{\mathbf{y}}$.

5) Apply the $N$-point DCT3e block: $\mathbf{Y}=\mathbf{C}_{3 e} \cdot \widetilde{\mathbf{y}}$

6) Compute $\widehat{\mathbf{H}}_{k}=\mathbf{Y}_{k} / d_{k}$ by means of the 1-tap per subcarrier coefficient obtained in Step 2.

7) Obtain $\widehat{\mathbf{h}}_{z p}=\mathbf{C}_{3 e}^{-1} \cdot \widehat{\mathbf{H}}$ by an $N$-point inverse DCT3e, which is the desired estimation of the zero padded channel filter, i.e., $\widehat{\mathbf{h}}_{z p}=[0, \widehat{\mathbf{h}}, 0, \ldots, 0]$.

\section{Numerical Results}

In this section, we test the behaviour of the proposed channel estimation scheme using one of the standardized ITUR M.1225 channels [16]. First of all, a length $N$ training signal, $\mathbf{X}$, is constructed in the DCT3e domain:

$$
X_{k}=2 \cos \left(\frac{\pi(2 k+1) M}{2 N}\right), \quad k=0,1, \ldots, N-1 .
$$

The order $N$ inverse DCT3e of $\mathbf{X}$ is performed, and the resulting length $N$ time-domain signal with WS symmetry, $\mathbf{x}$, is transmitted. ${ }^{1}$ After passing this signal through the channel,

\footnotetext{
${ }^{1}$ This process can be avoided simply by pre-computing $\mathbf{x}$ and storing it
} 


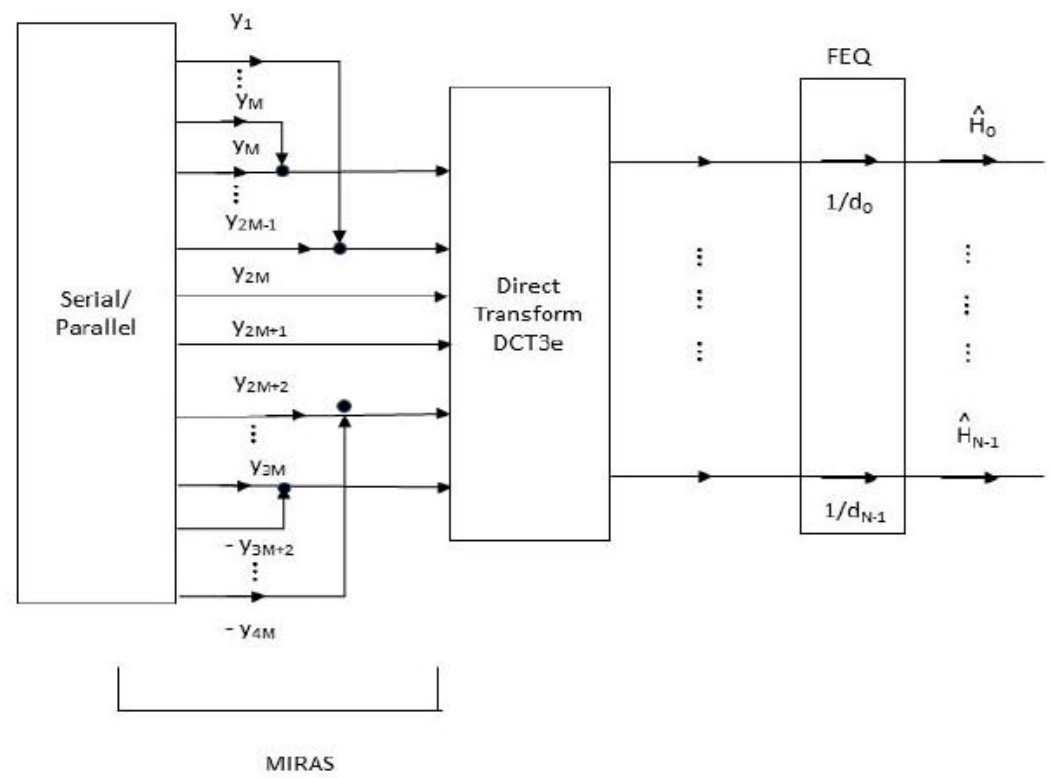

Fig. 3. MIRAS procedure at the receiver to compute $\tilde{\mathbf{y}}$, followed by a DCT3e to obtain $\mathbf{Y}$ and division by the coefficients $d_{k}$ to estimate the channel.
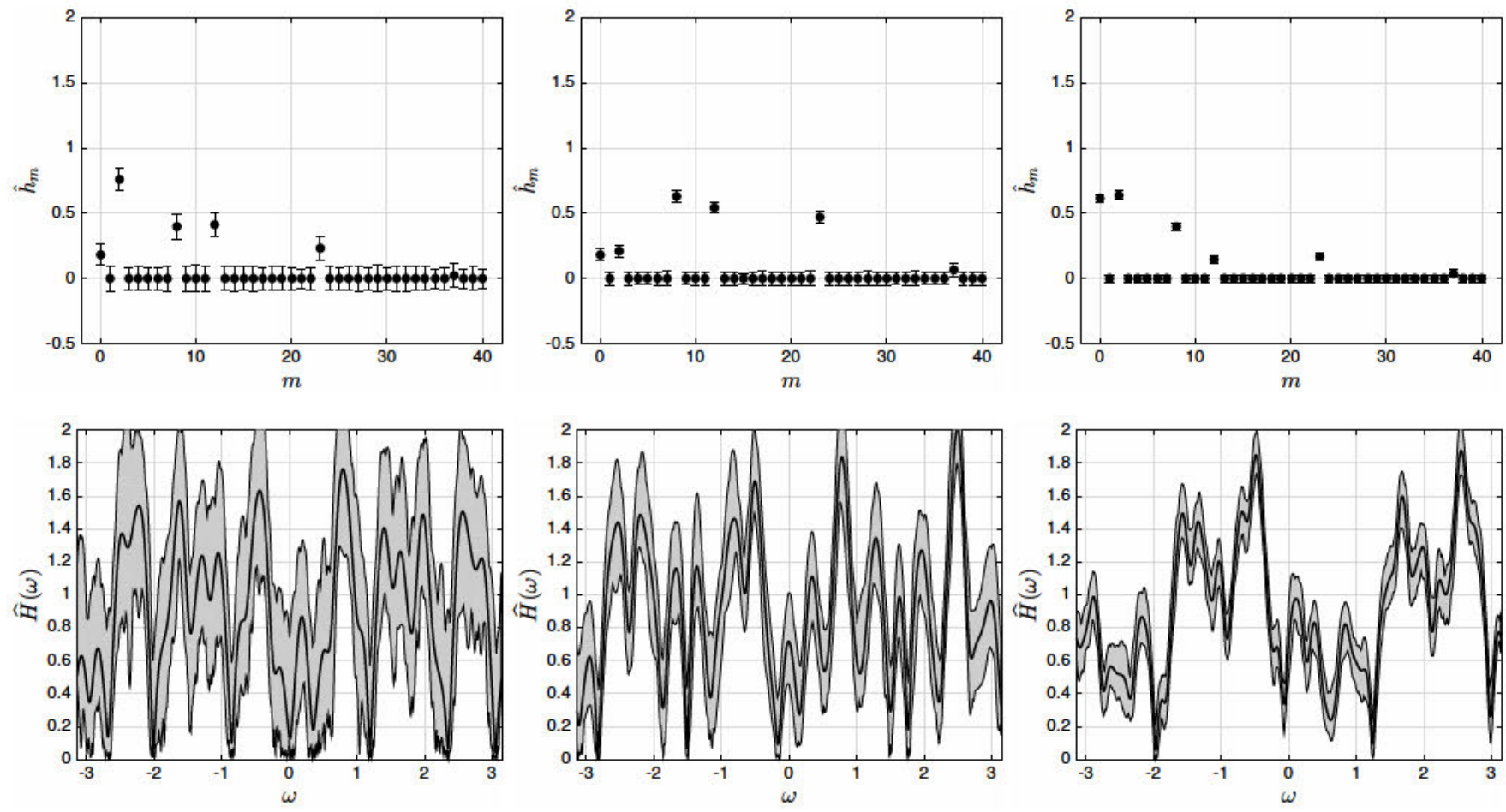

Fig. 4. ((a)-(c): Estimated channel's impulse response (true value and two times the standard deviation) for $\mathrm{SNR}=0 \mathrm{~dB}$ (a), $\mathrm{SNR}=5 \mathrm{~dB}$ (b), and $\mathrm{SNR}=$ $10 \mathrm{~dB}$ (c). (d)-(f): Estimated channel's frequency response (true value and range between maximum and minimum estimated values) for SNR $=0 \mathrm{~dB}$ (d), $\mathrm{SNR}=5 \mathrm{~dB}(\mathrm{e})$, and $\mathrm{SNR}=10 \mathrm{~dB}$ (f). In each case, an $N=1023$ length DCT3e was used and $N_{s}=100$ simulations were performed.

characterized by an impulse response vector of length $L, \mathbf{h}$, and adding a vector of independend and identically distributed zero-mean additive white Gaussian noise (AWGN) samples with variance $\sigma_{n}^{2}$, $\mathrm{z}$, we obtain the received signal vector of length $N+L-1, \mathrm{y}$. The MIRAS procedure described in Section II-B (see also Fig. 3) is then applied on the vector $\mathrm{z}$, thus obtaining the length $N=2 M+1$ vector $\widetilde{\mathbf{y}}$. Finally, its DCT3e of order $N$ is computed, Y, and a division by the pre-stored coefficients $d_{k}$ is performed to estimate the channel.

As mentioned above, we consider one of the channels standardized by ITU-R for the evaluation of radio transmission technologies for IMT 2000 [16]. More precisely, we address 
the estimation of the ITU-T M.1225 pedestrian channel B for $N=1023$. The channels were generated using Matlab's stdchan function using a carrier frequency $f_{c}=2 \mathrm{GHz}$ and a sampling period $T_{s}=100 \mathrm{~ns}$. With this sampling period, the channel's impulse response becomes $h_{m}=A_{0} \delta_{m}+A_{2} \delta_{m-2}+$ $A_{8} \delta_{m-8}+A_{12} \delta_{m-12}+A_{23} \delta_{m-23}+A_{37} \delta_{m-37}$, where each of the $A_{i}$ are independent Rayleigh distributed random variables and $\delta$ denotes Kronecker's delta. Note that the length of the channel's impulse response is actually $L^{\prime}=38$, but we set $L=41$ for the simulations in order to show the robustness of the proposed approach.

Fig. 4 shows three examples of the reconstructed channel (both in the time and frequency domains) for $N=1023$ and different SNRs. Note that the channel's reconstruction is reasonably good for SNR $=0 \mathrm{~dB}$ (Figs. 4(a) and 4(d)), it improves considerably for SNR $=5 \mathrm{~dB}$ (Figs. 4(b) and 4(e)), and it becomes almost perfect for SNR $=10 \mathrm{~dB}$ (Figs. 4(c) and 4(f)). Indeed, the reconstruction SNR (i.e., the ratio of the channel's impulse response power and the power of the reconstruction error) is $13.83 \mathrm{~dB}, 18.85 \mathrm{~dB}$ and 23.84 for an $\mathrm{SNR}=0 \mathrm{~dB}, \mathrm{SNR}=5 \mathrm{~dB}$ and $\mathrm{SNR}=10 \mathrm{~dB}$, respectively (i.e., approximately $13.84 \mathrm{~dB}$ higher than the SNR in the channel). Finally, note also that the impulse and frequency responses of the channels used in the two examples are different, since they have been generated randomly, as described before.

\section{CONCLUSIONS}

In this work, we have presented a general procedure for the estimation of any channel filter by means of the DCT3e. By using any training symbol with whole-sample (WS) symmetry, we show how to modify the received vector and how to take into account the information of the training signals to estimate the channel impulse response (CIR). The technique consists of including a "mirror, replicate and add/substract" (MIRAS) block processing at the receiver. Matrix formulation has been used to meet the conditions that guarantee perfect estimation of the CIR in the absence of noise. Our procedure allows estimation of the CIR without prior knowledge of its exact length, provided that it is shorter than the length of the symbol. If the channel's length is small enough, the procedure turns out to be a simple "mirror, replicate and add transformation". In any case, the channel is estimated by means of one-tap coefficients in the transform domain that are also computed via the DCT3e. Simulations show that the proposed algorithm attains very accurate channel estimates in noisy environments for the ITU-T M.1225 pedestrian channel B. Future research lines include developing blind and semi-blind channel estimation procedures, addressing the case where neither the training signal nor the channel are symmetric, and considering the joint estimation of MIMO channels for DCT-based multicarrier modulation (MCM).

\section{ACKNOWLEDGMENT}

This work has been partially supported by the Spanish Ministry of Economy and Competitiveness through project TEC2015-64835-C3-3-R. The authors are members of the
TACA Research Group of the Universidad Politécnica de Madrid (Spain) and would like to thank UPM for its support.

\section{REFERENCES}

[1] N. Al-Dhahir, H. Minn, S. Satish, "Optimum DCT-based multicarrier transceivers for frequency-selective channels", IEEE Transactions on Communications, vol. 54, no. 5, pp. 911-921, May 2006.

[2] P. Tan and N.C. Beaulieu, "A comparison of DCT-based OFDM and DFT-based OFDM in frequency offset and fading channels", IEEE Transactions on Commununications, vol. 54, no. 11, pp. 2113-2125, Nov. 2006.

[3] F. Cruz-Roldán, M. E. Domínguez-Jiménez, G. Sansigre-Vidal, P. AmoLópez, M. Blanco-Velasco, and Á. Bravo-Santos, "On the use of discrete cosine transforms for multicarrier communications," IEEE Transactions on Signal Processing, vol. 60, no. 11, pp. 6085-6090, Nov. 2012.

[4] P. Kumar, P. Kumar, "Performance evaluation of modified OFDM for underwater communications", 2013 IEEE International Conference on Communications Workshops (ICC), pp. 967-971, 9-13 June 2013.

[5] B. Mathew, P. George, R. V. Nathan, S Shukkor, A. N. Lakshmi, "BER comparison of DCT and FFT based OFDM systems in AWGN and Rayleigh fading channels with different modulation schemes", 2013 Annual International Conference on Emerging Research Areas and 2013 International Conference on Microelectronics, Communications and Renewable Energy (AICERA/ICMiCR), pp. 1-4, 4-6 June 2013.

[6] Y. Liu, Z. Tan, H. Hu, L. J. Cimini, G. Y. Li, "Channel estimation for OFDM, " IEEE Communications Surveys \& Tutorials, vol. 16, no. 4, pp. 1891-1908, 2014.

[7] F. Zabini, B. M. Masini, A. Conti, L. Hanzo, "Partial equalization for MC-CDMA systems in non-ideally estimated correlated fading. "IEEE Transactions on Vehicular technology, vol. 59, no. 8, pp. 3818-3830, 2010.

[8] F. Zabini, G. Pasolini, O. Andrisano, "Design criteria for FIR-based echo cancellers, "IEEE Transactions on Broadcasting, vol. 62, no. 3, pp. 562-578, 2016.

[9] Z. Zheng, F. Frey, P. W. Berenguer, J. K. Fischer, "Low-Complexity Equalization Scheme for Multicarrier Offset-QAM Systems, "IEEE Photonics Technology Letters, vol. 29, no. 23, pp. 2075-2078, 2017.

[10] S. Peng, A. Liu, X. Liu, K. Wang, X. Liang, "MMSE Turbo Equalization and Detection for Multicarrier Faster-Than-Nyquist Signaling, "IEEE Transactions on Vehicular Technology, vol. 67, no. 3, pp. 2267-2275, 2018.

[11] F. Cruz-Roldán, M. E. Domínguez-Jiménez, G. Sansigre-Vidal, D. Luengo, and M. Moonen, "DCT-based channel estimation for singleand multicarrier communications," Signal Processing., vol. 128, pp. 332339, Nov. 2016.

[12] M. E. Domínguez-Jiménez, D. Luengo, and G. Sansigre-Vidal, "Estimation of symmetric channels for discrete cosine transform Type-I multicarrier systems: A compressed sensing approach" The Scientific World Journal, pp. 1-11, 2015.

[13] M. E. Domínguez-Jiménez, D. Luengo, G. Sansigre-Vidal and F. CruzRoldán, "A Novel Channel Estimation Scheme for Multicarrier Communications with the Type-I even Discrete Cosine Transform ," Proceedings of the 25th European Signal Processing Conference (EUSIPCO 2017), pp. 2303-2307, Sept. 2017.

[14] S. A. Martucci, "Symmetric convolution and the discrete sine and cosine transforms”, IEEE Transactions on Signal Processing, vol. 42, no. 5, pp. 1038-1051, May 1994.

[15] V. Sánchez, P. García, A. M. Peinado, J. C. Segura, and A. J. Rubio, "Diagonalizing properties of the discrete cosine transforms" IEEE Transactions on Signal Processing, vol. 43, no. 11, pp. 2631-2641, Nov. 1995.

[16] Recommendation ITU-R M.1225, "Guidelines for Evaluation of Radio Transmission Technologies for IMT-2000”, 1997. 